\title{
Development of New Probiotics by Strain Combinations: Is It Possible to Improve the Adhesion to Intestinal Mucus?
}

\author{
M. C. Collado, ${ }^{* 1}$ J. Meriluoto, $†$ and S. Salminen* \\ *Functional Foods Forum, University of Turku, Itäinen Pitkäkatu 4A, 20520 Turku, Finland \\ †Department of Biochemistry and Pharmacy, Åbo Akademi University, Tykistökatu 6A, 20520 Turku, Finland
}

\begin{abstract}
We evaluated the ability of commercial probiotic strains (Lactobacillus rhamnosus GG, L. rhamnosus LC705, Bifidobacterium breve 99, and Propionibacterium freudenreichii ssp. shermanii JS) to adhere alone or in different combinations to immobilized mucus. Probiotic combinations were clearly able to enhance the adhesion of L. rhamnosus GG, L. rhamnosus LC705, and $P$. freudenreichii ssp. shermanii JS. For L. rhamnosus GG and $P$. freudenreichii JS, all the combinations significantly improved adhesion to intestinal mucus, from 29.7 to $34.9 \%$ and from 1.9 to $2.3 \%$, respectively. The adhesion of $L$. rhamnosus LC705 was improved from 0 to $46.4 \%$. The adhesion of $B$. breve 99 was improved only in combination with $L$. rhamnosus GG and $P$. freudenreichii JS. Our results suggest that probiotic combinations could increase the beneficial health effects as compared with individual strains. Combinations of probiotic strains may therefore have synergistic adhesion effects, and such combinations also should be assessed in clinical studies.
\end{abstract}

Key words: adhesion, probiotic combinations, synergy

\section{INTRODUCTION}

A probiotic has been defined as a "live microorganism which, when administered in adequate amounts, confers a health benefit on the host" (FAO/WHO, 2002). Selected strains, belonging mostly to the Lactobacillus and Bifidobacterium genera, but also some Propionibacterium strains, have been introduced as probiotics in food products because of their health-promoting effects. Among the important criteria suggested for selection of probiotics is the ability to adhere to the gastrointestinal mucosa and competitively exclude the most frequently considered pathogens (Salminen et al., 1999; Ouwehand et al., 2002). Adhesion and colonization of the

Received July 19, 2006.

Accepted February 19, 2007.

${ }^{1}$ Corresponding author: marcol@utu.fi mucosal surfaces by probiotics are possible protective mechanisms against pathogens through competition for binding sites and nutrients (Ouwehand et al., 2002; Collado et al., 2005) or immune modulation (Salminen et al., 1999). Adhesion is also regarded as a prerequisite for colonization (Beachey, 1981). In spite of the lack of definitive proof, some studies have indicated a relationship between in vitro adhesion and in vivo colonization (Crociani et al., 1995; Cesena et al., 2001).

Probiotic strains have been shown to exert a protective effect against acute diarrhea, rotavirus diarrhea, and antibiotic-associated diarrhea (Chouraqui et al., 2004; Gaudier et al., 2005; McFarland, 2006; Santosa et al., 2006; Sazawal et al., 2006; Zocco et al., 2006), as well as Helicobacter pylori infection (Gotteland et al., 2006), and they alleviate symptoms of gastrointestinal diseases such as irritable bowel syndrome (Kajander et al., 2005; Kim et al., 2005; Camilleri, 2006). In addition, probiotics have shown other health benefits related to pathogen infection and immune system stimulation (Reid and Hammond, 2005; Rautava et al., 2006; Santosa et al., 2006). An increasing number of scientific reports have appeared on the effects of probiotic combinations on the health of the host (Femia et al., 2002; Gionchetti et al., 2005; Kajander et al., 2005; Myllyluoma et al., 2005; Olivares et al., 2006; Roselli et al., 2006). Research using single probiotic strains has been reported earlier, but at present, probiotic combinations with possible additional health benefits are being assessed prior to use in clinical studies. The best known probiotic combination analyzed in the last several years, consisting of a mixture of 8 lactic acid bacterial species (VSL\#3), has been reported to be effective in several human diseases (Bibiloni et al., 2005; Gaudier et al., 2005; Gionchetti et al., 2005; Kim et al., 2005; Camilleri, 2006).

For instance, adhesion in vivo could be influenced by both the normal microbiota and the specific probiotics included in each preparation. However, few studies are available on the adhesion interactions of probiotics in the intestinal mucus system (Ouwehand et al., 2000). We hypothesized that combinations of adherent probiotic strains would influence the adhesion of individual 
strains in the human intestinal mucus, either enhancing or decreasing the adhesion. The aim of this study was to characterize the adhesion of Lactobacillus rhamnosus GG, L. rhamnosus LC705, Bifidobacterium breve 99, and Propionibacterium freudenreichii ssp. shermanii JS strains, either alone or in different combinations, to human intestinal mucus in the immobilized human intestinal mucus model. Good correlations have previously been reported between this model and the enterocyte-like Caco-2 model (Aissi et al., 2001; Gueimonde et al., 2006).

\section{MATERIALS AND METHODS}

\section{Bacterial Strains and Culture Conditions}

The commercial lactic acid bacteria strains used in this study were $L$. rhamnosus GG (ATCC 53103), $L$. rhamnosus LC705, B. breve 99, and P. freudenreichii ssp. shermanii JS. All the strains were kindly provided by Valio Ltd. (Helsinki, Finland) and were stored in the Functional Foods Forum Culture Collection, University of Turku, Finland.

For assays, lactobacilli were cultured in de Man, Rogosa, Sharpe broth; bifidobacteria were cultured in de Man, Rogosa, Sharpe broth (Scharlau Chemie, Barcelona, Spain) with $0.05 \%$ (wt/vol) Cys-HCl; and propionibacteria were grown in Gifu anaerobic medium (GAM Nissui Pharmaceutical, Tokyo, Japan). All microorganisms were metabolically labeled by addition to the media of $10 \mu \mathrm{L} / \mathrm{mL}$ of tritiated thymidine $\left(5-{ }^{3} \mathrm{H}\right.$-thymidine, $1.0 \mathrm{mCi} / \mathrm{mL}$; Amersham Biosciences, Little Chalfont, UK) and were incubated overnight at $37^{\circ} \mathrm{C}$ under anaerobic conditions $\left(10 \% \mathrm{H}_{2}, 10 \% \mathrm{CO}_{2}\right.$, and $80 \% \mathrm{~N}_{2}$; Concept 400 anaerobic chamber, Ruskinn Technology, Leeds, UK),

Radiolabeled bacteria were then harvested and washed twice with PBS buffer (130 m $M$ sodium chloride, $10 \mathrm{~m} M$ sodium phosphate, $\mathrm{pH} 7.2$ ), and the absorbance $\left(\mathrm{A}_{600 \mathrm{~nm}}\right)$ was adjusted to $0.25 \pm 0.05$ to standardize the bacterial concentration (approximately $10^{8}$ $\mathrm{cfu} / \mathrm{mL}$ ) of each strain. Bacterial suspensions of probiotic combinations were made by mixing equal amounts of each probiotic strain $(1: 1 \mathrm{vol} / \mathrm{vol}$ in a 2 strain combination, 1:1:1 vol/vol in a 3-strain combination, and 1:1:1:1 in a 4-strain combination), but only a single probiotic strain was labeled to detect the influence of the presence of the other probiotic strains in combination.

\section{In Vitro Adhesion Assay to Intestinal Human Mucus}

Human intestinal mucus was collected from the healthy part of resected colonic tissue as described by Ouwehand et al. (2002) and dissolved (0.5 mg of protein/
$\mathrm{mL}$ ) in HEPES-Hanks buffer (HH; $10 \mathrm{~m} M$ HEPES, $\mathrm{pH}$ 7.4). One hundred microliters of the mucus solution was immobilized in polystyrene microtiter plate wells (Maxisorp, Nunc, Denmark) by overnight incubation at $4^{\circ} \mathrm{C}$.

The adhesion assessment was carried out as previously described (Collado et al., 2005). Radiolabeled bacteria were harvested after overnight incubation and washed twice with $\mathrm{HH}$ buffer. Absorbance $\left(\mathrm{A}_{600 \mathrm{~nm}}\right)$ was adjusted to $0.25 \pm 0.05$ to standardize the number of bacteria (approximately $10^{8} \mathrm{cfu} / \mathrm{mL}$ ), and $100 \mu \mathrm{L}$ of this suspension was added to the wells and incubated for 1 $\mathrm{h}$ at $37^{\circ} \mathrm{C}$. Subsequently, the wells were washed twice with $200 \mu \mathrm{L}$ of $\mathrm{HH}$ to remove unattached bacteria. Adhered bacteria were released and lysed with $1 \%$ (wt/ vol) SDS in $0.1 \mathrm{~mol} / \mathrm{L}$ of $\mathrm{NaOH}(200 \mu \mathrm{L}$ per well) by incubation at $65^{\circ} \mathrm{C}$ for $1 \mathrm{~h}$. The radioactivity of the lysed suspension was measured by liquid scintillation (OptiPhase HiSafe 3; Wallac Oy, Turku, Finland) with a 1450 Microbeta liquid scintillation counter (Wallac Oy). Adhesion was calculated as the percentage of radioactivity recovered after adhesion relative to the radioactivity of the bacterial suspension added to the mucus.

\section{Coaggregation Assays with Probiotic Strains}

The coaggregation test was performed as described earlier (Handley et al., 1987). Briefly, overnight cultures were harvested by centrifugation at $3,200 \times g$ and $4^{\circ} \mathrm{C}$ for $10 \mathrm{~min}$, washed 3 times with PBS buffer, and suspended in the same buffer. Absorbances $\left(\mathrm{A}_{600 \mathrm{~nm}}\right)$ were then adjusted to $0.25 \pm 0.05$ to standardize the number of bacteria (approximately $10^{8} \mathrm{cfu} / \mathrm{mL}$ ). Each probiotic strain combination suspension was incubated at $20^{\circ} \mathrm{C}$ and suspensions were then monitored during $4 \mathrm{~h}$ of incubation. To prepare the combination, equal volumes of bacterial cells of the different probiotic strains (1:1 vol/vol in a 2-strain combination; $1: 1: 1 \mathrm{vol} /$ vol in a 3 -strain combination, and 1:1:1:1 in a 4-strain combination) were prepared and mixed and incubated at $20^{\circ} \mathrm{C}$ without agitation. Percentages of aggregation were calculated using the formula $\left[\left(\mathrm{A}_{\text {time }}-\mathrm{A}_{\text {mix }}\right) / \mathrm{A}_{\text {time }}\right]$ $\times 100$, where $A_{\text {time }}$ represents the absorbance of the mix containing the probiotic combinations at time $0 \mathrm{~h}$, and $\mathrm{A}_{\text {mix }}$ represents the absorbance of the mixed culture at different incubation times.

\section{Statistical Analysis}

Statistical analysis was done using the SPSS 11.0 software (SPSS Inc., Chicago, IL). The results were expressed as the average of 4 independent experiments. Each experiment was performed with 3 parallels. A 
2 -tailed $t$-test for unpaired observations was used to evaluate the statistical significance $(P<0.05)$ of the differences in the ability of each bacterial strain to adhere in the presence or absence of another bacterial strain.

\section{RESULTS}

\section{In Vitro Adhesion Assay to Intestinal Human Mucus}

All probiotic strains tested were able to adhere to intestinal mucus. The percentages, expressed as mean \pm standard deviation, were $20.0 \% \pm 2.0$ for $L$. rhamnosus GG, $1.2 \% \pm 0.7$ for L. rhamnosus LC705, $2.5 \% \pm 0.3$ for $B$. breve 99, and $0.9 \% \pm 0.5$ for $P$. freudenreichii JS. All combinations increased the adhesion levels of $L$. rhamnosus GG, L. rhamnosus LC705, and P. freudenreichii JS but, interestingly, these improvements were not detected for $B$. breve 99. Bifidobacterium breve 99 in all possible combination (2-, 3-, and 4-strain combinations with GG, LC705, and JS) showed a significant ( $P$ $<0.05$ ) reduction in adhesion, but only one combination was able to improve adhesion. This combination was $B$. breve $99, L$. rhamnosus $\mathrm{GG}$, and $P$. freudenreichii JS. The changes to intestinal mucus in the adhesion of each strain in combination with other probiotics are shown in Figure 1.

For L. rhamnosus GG $(20.0 \% \pm 2.0$ adhesion $)$ and $P$. freudenreichii JS $(0.9 \% \pm 0.5$ adhesion $)$, all the combinations significantly $(P<0.05)$ improved adhesion to intestinal mucus, from $29.7 \% \pm 1.5$ to $34.9 \% \pm 3.5$ and from $1.9 \% \pm 0.3$ to $2.3 \% \pm 0.2$, respectively, in absolute values. The changes in the adhesion values, taken as $0 \%$ adhesion of each single strain, were from 47.4 to $72.9 \%$ for L. rhamnosus GG and from 132.4 to $157.9 \%$ for P. freudenreichii.

The adhesion value of $L$. rhamnosus LC705 (1.2\%) in combination with other probiotic strains was improved, with values ranging from $1.2 \% \pm 0.0$ to $1.7 \% \pm 0.4$. The adherence of $L$. rhamnosus LC705 was found to be significantly $(P<0.05)$ higher in combination with $L$. rhamnosus GG, by $46.4 \%$ ( $1.7 \% \pm 0.3$ adhesion). Furthermore, in combination with $L$. rhamnosus $\mathrm{GG}$ and $P$. freudenreichii JS, the adhesion of $L$. rhamnosus LC705 was enhanced by $31.0 \%$ (1.6\% \pm 0.4 adhesion, $P<0.05)$, and in combination with $P$. freudenreichii JS and $B$. breve 99 was enhanced by $33.5 \%$ (1.6\% \pm 0.3 adhesion, $P<0.05)$. A combination of all 3 strains improved the adhesion of $L$. rhamnosus LC705 by $45.3 \%$ (1.7\% \pm 0.3 , $P<0.05)$. The adhesion of $B$. breve $99(2.5 \% \pm 0.3)$ was improved only in combination with $L$. rhamnosus GG and $P$. freudenreichii JS $(2.7 \% \pm 0.2, P>0.05)$, whereas the other combinations did not enhance the adhesion of $B$. breve 99 .
To significantly improve the adhesion of L. rhamnosus GG to intestinal mucus, the best combination was with $L$. rhamnosus LC705 and P. freudenreichii JS. For the adhesion of $L$. rhamnosus LC705, the best combination was with $L$. rhamnosus GG, whereas for P. freudenreichii JS, the optimal combination was with 2 strains, $L$. rhamnosus GG and $B$. breve 99. Interestingly, only one combination (B. breve 99-L. rhamnosus GG- $L$. rhamnosus LC705) was able to improve the adhesion of $B$. breve 99 (7.5\%), although in a nonsignificant manner $(P>0.05)$.

\section{Coaggregation Assays with Probiotic Strains}

Because of the adhesion effect observed with different combinations of the probiotic strains tested, we wanted to know whether there were coaggregation abilities within the strains and whether these abilities could enhance or decrease the adhesion properties. The coaggregation properties were calculated according to the procedure described by Handley et al. (1987). The results of coaggregation abilities are shown in Table 1. All probiotic combinations tested showed aggregation abilities, but the percentage of aggregation was demonstrated to be strain-specific and dependent on time conditions. In general, the aggregation abilities of each strain alone showed higher percentages of aggregation than each strain in different combinations, mainly during the first hours of incubation. The highest coaggregative combination of probiotic strains was obtained with L. rhamnosus LC705 and GG $(14.3 \pm 1.3 \%)$ after $4 \mathrm{~h}$ of incubation at room temperature. The addition of $P$. freudenreichii JS and B. breve 99 to the combination of L. rhamnosus LC705 and GG did not decrease their coaggregation $(P>0.05 ; 10.6 \pm 1.5 \%$ and $10.9 \pm 1.2 \%$, respectively). In addition, the combinations $P$. freudenreichii JS-L. rhamnosus LC705 and $P$. freudenreichii JS-L. rhamnosus GG showed good coaggregation abilities, with percentages higher than $10 \%(P<0.05)$.

\section{DISCUSSION}

Bacterial adhesion is a complex process involving contact of the cell with the surface, and it is affected by the composition and structure of the cell membrane and interacting surfaces. The ability to adhere to epithelial cells and mucosal surfaces has been suggested to be an important property of many bacterial strains used as probiotics. Several researchers have reported results on the composition, structure, and forces of interaction related to bacterial adhesion to intestinal epithelial cells (Pérez et al., 1998; Del Re et al., 2000) and mucus (Tuomola et al., 2000; Collado et al., 2005). In most cases, aggregation ability is related to cell adherence 
A
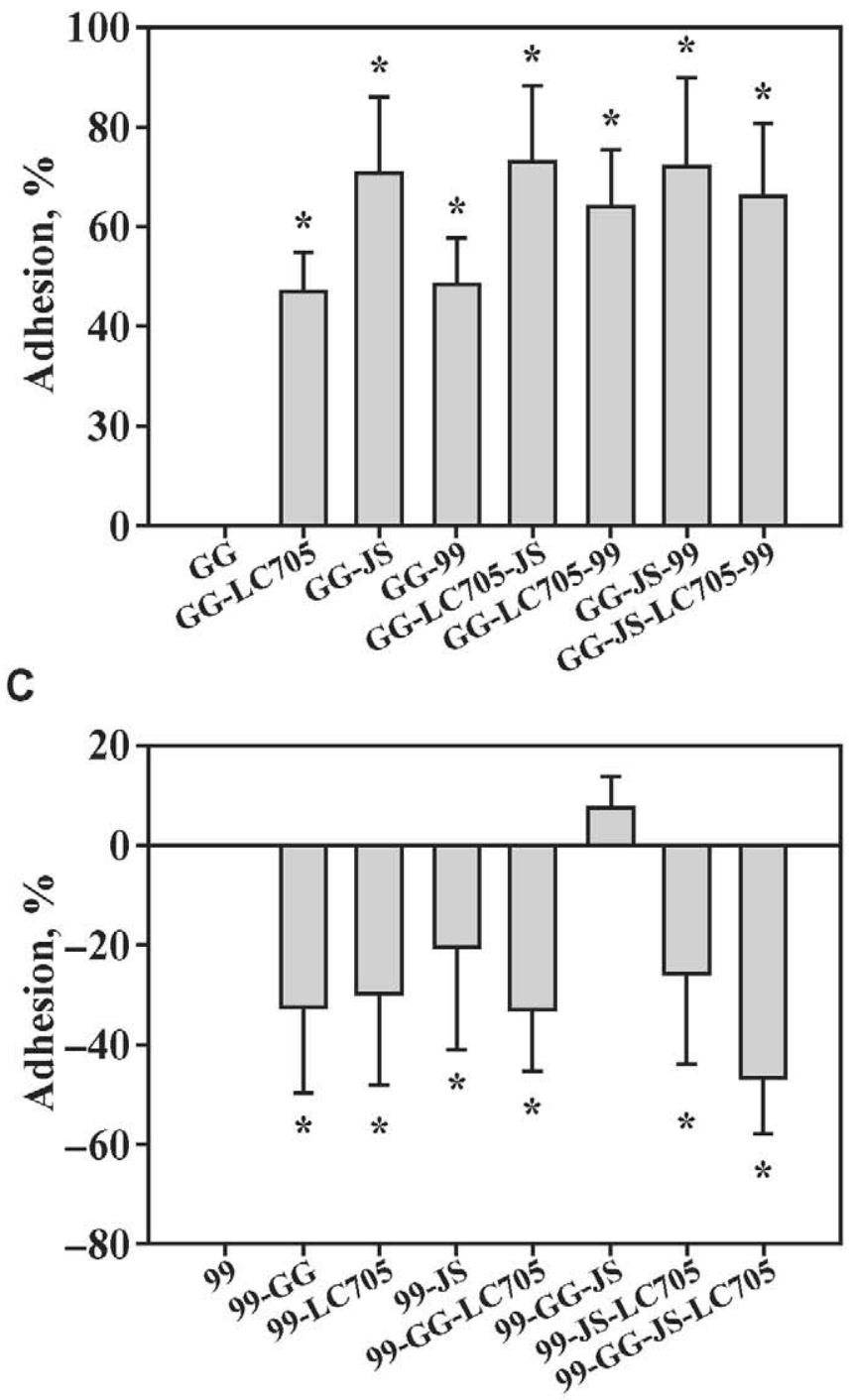

Strain
B
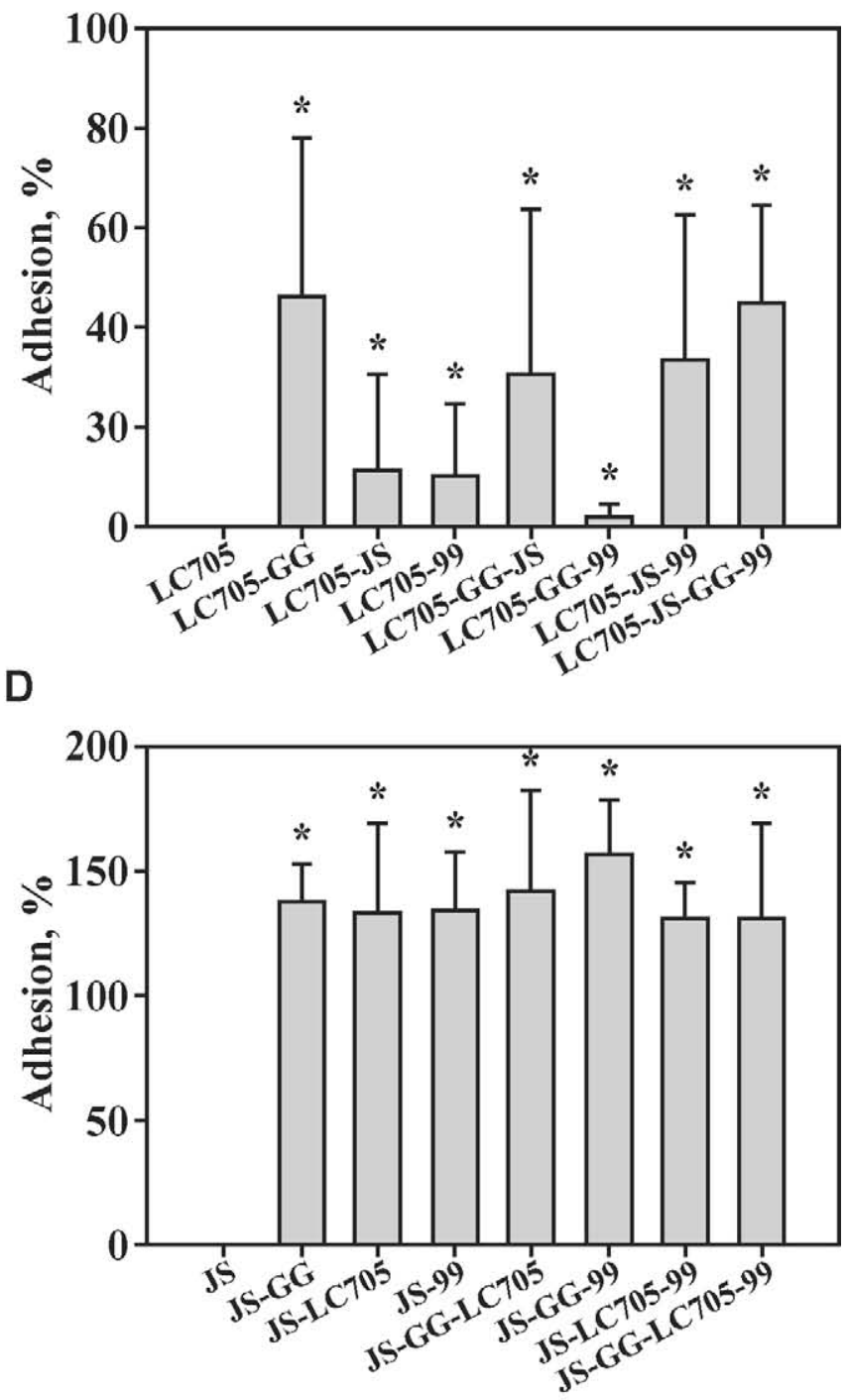

Strain

Figure 1. Changes in adhesion percentages of (A) Lactobacillus rhamnosus GG, (B) L. rhamnosus LC705, (C) Bifidobacterium breve 99, and (D) Propionibacterium freudenrenchii JS in 2-, 3-, and 4-strain combinations. Adhesion of each strain alone is taken as 0\%. An asterisk (*) indicates statistical significance $(P<0.05)$ compared with the control $(0 \%$ adhesion). Bacteria radiolabeled in each experiment were: $(\mathrm{A})$ L. rhamnosus GG; (B) L. rhamnosus LC705; (C) B. breve 99; and (D) P. freudenrenchii JS. The other bacteria were not labeled.

properties (Boris et al., 1997; Del Re et al., 2000). However, it can be hypothesized that a combination of probiotic strains may complement or improve the health benefits given by individual strains. In in vitro trials, the probiotic properties have mainly been tested alone or in combination with yogurt bacteria such as $L$. delbrueckii and L. acidophilus (Ouwehand et al., 2000) but have rarely been combined with other probiotics. In addition, a few in vivo studies have used probiotic combinations, and further health benefits have been reported with LGG, PJS, LC705, and B. breve 99 (Kajander et al.,
2005; Myllyluoma et al., 2005) for VSL\#3 (Bibiloni et al., 2005; Gaudier et al., 2005; Gionchetti et al., 2005; Kim et al., 2005; Camilleri, 2006).

However, few studies are available on the interactions of probiotics regarding adhesion properties in the intestinal mucus system (Ouwehand et al., 2000). In addition, a recent report (Ruas-Madiedo et al., 2006) describes how the presence of exopolysaccharides produced by probiotic bacteria can modify bacterial adhesion to the intestinal mucus. For all these, the objective of the present study was to determine whether the ad- 
Table 1. Coaggregation abilities of Lactobacillus rhamnosus GG, L. rhamnosus LC705, Propionibacterium freudenrenchii JS, and Bifidobacterium breve 99 in different combinations during incubation at $20^{\circ} \mathrm{C}^{1}$

\begin{tabular}{lcrrr}
\hline \multirow{2}{*}{$\begin{array}{l}\text { Strains and } \\
\text { combinations }\end{array}$} & \multicolumn{4}{c}{ Aggregation, \% } \\
\cline { 2 - 5 } & 1-h incubation & 2-h incubation & 3 -h incubation & 4-h incubation \\
\hline GG & $4.0 \pm 1.0^{*}$ & $4.6 \pm 1.2^{*}$ & $5.2 \pm 1.0^{*}$ & $6.0 \pm 1.1^{*}$ \\
LC705 & $9.2 \pm 4.0^{*}$ & $20.4 \pm 10.5^{*}$ & $26.8 \pm 1.2^{*}$ & $27.0 \pm 2.0^{*}$ \\
JS & $3.2 \pm 1.1^{*}$ & $7.7 \pm 2.1^{*}$ & $8.0 \pm 2.6^{*}$ & $8.2 \pm 1.2^{*}$ \\
99 & $5.5 \pm 2.1^{*}$ & $12.6 \pm 1.1^{*}$ & $12.8 \pm 1.0^{*}$ & $13.0 \pm 0.7^{*}$ \\
JS-LC705 & $4.1 \pm 3.5$ & $6.9 \pm 2.5^{*}$ & $9.9 \pm 1.9^{*}$ & $13.0 \pm 0.7^{*}$ \\
JS-99 & $0.9 \pm 0.1$ & $3.4 \pm 1.2$ & $5.6 \pm 1.8^{*}$ & $7.9 \pm 2.3^{*}$ \\
JS-GG & $1.8 \pm 1.8$ & $4.4 \pm 1.8^{*}$ & $8.2 \pm 0.2^{*}$ & $10.5 \pm 0.7^{*}$ \\
99-LC705 & $0.1 \pm 0.1$ & $3.4 \pm 1.8$ & $6.3 \pm 1.5^{*}$ & $9.1 \pm 1.5^{*}$ \\
LC705-GG & $0.7 \pm 1.2$ & $4.6 \pm 3.1$ & $9.2 \pm 3.7^{*}$ & $14.3 \pm 1.3^{*}$ \\
99-GG & $0.4 \pm 1.3$ & $3.6 \pm 1.9$ & $6.1 \pm 0.2^{*}$ & $9.2 \pm 2.3^{*}$ \\
JS-LC705-99 & $0.4 \pm 0.9$ & $3.6 \pm 2.4$ & $6.7 \pm 0.8^{*}$ & $9.4 \pm 1.6^{*}$ \\
LC705-JS-GG & $1.5 \pm 1.3$ & $4.8 \pm 3.0$ & $8.8 \pm 2.6^{*}$ & $10.6 \pm 1.5^{*}$ \\
LC705-99-GG & $0.9 \pm 1.4$ & $4.7 \pm 1.5^{*}$ & $7.8 \pm 1.6^{*}$ & $10.9 \pm 1.2^{*}$ \\
JS-99-GG & $0.6 \pm 0.5$ & $3.9 \pm 2.4$ & $6.3 \pm 0.7^{*}$ & $8.9 \pm 1.7^{*}$ \\
LC705-99-GG-JS & $0.9 \pm 0.6$ & $6.9 \pm 6.8$ & $10.1 \pm 6.6^{*}$ & $12.8 \pm 4.6^{*}$ \\
\hline
\end{tabular}

${ }^{1}$ Initial absorbance $\left(\mathrm{A}_{600 \mathrm{~nm}}\right)$ at time 0 was approximately $0.25 \pm 0.05$.

*Differences $(P<0.05)$, compared with the control taken as $0 \%$ (no aggregation).

hesion of a probiotic strain would be affected by the presence of other probiotics. This is necessary to test future probiotic combinations and to find the best combinations to be included in fermented milk products. All probiotic strains included in this study have documented health effects (Kajander et al., 2005; Myllyluoma et al., 2005). The adhesion levels of the tested commercial probiotic strains showed great variability depending on the strain, species, and genus. All strains tested were able to adhere in the model system used in this study; these results are in agreement with earlier observations (Tuomola et al., 2000; Collado et al., 2005; Gueimonde et al., 2006). All combinations (double, triple, and quadruple combinations) among the 4 probiotic strains tested in this study enhanced the adhesion of all probiotic strains. However, our study showed that $B$. breve 99 was an exception because only one combination improved its adhesion, but not significantly. This combination was $B$. breve $99, L$. rhamnosus GG, and $P$. freudenreichii JS.

In general, no correlations between adhesion and aggregation abilities were found among the probiotic combinations, because the highest increases in adhesion were not observed for the highest aggregative combinations. The most autoaggregative strain alone was $L$. rhamnosus LC705, but it was not the best strain to adhere to intestinal mucus because it showed only $1.2 \%$ of adhesion to mucus. In addition, the strain combinations with $L$. rhamnosus LC705 were not the most coaggregative combinations, suggesting that other mechanisms are related to aggregation abilities with other bacterial surfaces from different strains. The most coaggregative combination was $L$. rhamnosus GG with $L$. rhamnosus LC705 (14.3\%), but the same combination was not the best for adhesion of L. rhamnosus GG (46.4\%). The best combination for $L$. rhamnosus GG adhesion was in combination with L. rhamnosus LC705 and $P$. freudenreichii JS (72.9\%). We suggest that the greater increases in adhesion of L. rhamnosus GG and $P$. freudenreichii JS might be due to coaggregation with the other probiotic strains tested, and this was confirmed by our results, because they showed coaggregation abilities of more than $10 \%$. Therefore, the mechanism of the observed increase in binding remains unclear.

Our results suggest that probiotic combinations could increase the beneficial health effects as compared with individual strains. Adhesion properties influence colonization, modulation of the immune system, and protective mechanisms against infection by pathogens, and good adhesion exerts a protective effect against gastrointestinal disorders. By knowing that the health effects of probiotics are genus-, species-, and strain-specific (Timmerman et al., 2004), it is feasible that combinations of different probiotic strains may prove more effective than monostrain probiotics. Only a few studies have demonstrated that specific combinations of probiotics have enhanced the beneficial effects, compared with single probiotics (Zoppi et al., 2001; Gionchetti et al., 2002; Perdigon et al., 2002; Timmerman et al., 2004). Recent reports have described the health-promoting effects of probiotic combinations, including some strains tested in this study, in irritable bowel syndrome (Kajander et al., 2005), and against $H$. pylori infection (Myllyluoma et al., 2005).

Taken together, combining probiotic strains may result in enhanced adherence and coaggregation properties. It can therefore be hypothesized that a specific 
combination of these microorganisms may have the potential to increase the efficacy of probiotic preparations, but the result depends on the aberrancy of the target microbiota, the adhering organism, and its suitability to counteract the microbiota aberrancy in question. In the best cases, the strains may complement each other's probiotic effect and even work synergistically. We emphasize the fact that combinations of probiotic microorganisms should be tested in vitro prior to introducing such combinations in clinical intervention studies.

\section{ACKNOWLEDGMENTS}

This work was supported by the Academy of Finland, Research Council for Biosciences and Environment (decision number 210309 to Åbo Akademi and 210310 to University of Turku). M. C. Collado is the recipient of a Postdoctoral Excellence grant from Conselleria Empresa, Universidad y Ciencia de la Generalitat, Valenciana, Spain (BPOSTDOC 06/016). Valio Ltd. (Helsinki, Finland) provided the probiotic mixture used in the study.

\section{REFERENCES}

Aissi, E. A., M. Lecocq, C. Brassart, and S. Buoquelet. 2001. Adhesion of some Bifidobacteria strains to human enterocyte-like cells and binding to mucosal glycoproteins. Microb. Ecol. Health Dis. 13:32-39.

Beachey, E. H. 1981. Bacterial adherence: Adhesin-receptor interactions mediating the attachment of bacteria to mucosal surfaces. J. Infect. Dis. 143:325-345.

Bibiloni, R., R. N. Fedorak, G. W. Tannock, K. L. Madsen, P. Gionchetti, M. Campieri, C. De Simone, and R. B. Sartor. 2005. VSL\#3 probiotic-mixture induces remission in patients with active ulcerative colitis. Am. J. Gastroenterol. 100:1539-1546.

Boris, S., J. E. Suarez, and C. Barbes. 1997. Characterization of the aggregation promoting factor from Lactobacillus gasseri, a vaginal isolate. J. Appl. Microbiol. 83:413-420.

Camilleri, M. 2006. Probiotics and irritable bowel syndrome: Rationale, putative mechanisms, and evidence of clinical efficacy. J. Clin. Gastroenterol. 40:264-269.

Cesena, C., L. Morelli, M. Alander, T. Siljander, E. Tuomola, S. Salminen, T. Mattila-Sandholm, T. Vilpponen-Salmela, and A. Von Wright. 2001. Lactobacillus crispatus and its nonaggregating mutant in human colonization trials. J. Dairy Sci. 84:1001-1010.

Chouraqui, J. P., L. D. Van Egroo, and M. C. Fichot. 2004. Acidified milk formula supplemented with Bifidobacterium lactis: Impact on infant diarrhea in residential care settings. J. Pediatr. Gastroenterol. Nutr. 38:288-292.

Collado, M. C., M. Gueimonde, M. Hernandez, Y. Sanz, and S. Salminen. 2005. Adhesion of selected Bifidobacterium strains to human intestinal mucus and the role of adhesion in enteropathogen exclusion. J. Food Prot. 68:2672-2678.

Crociani, J., J.-P. Grill, M. Huppert, and J. Ballonge. 1995. Adhesion of different bifidobacteria strains to human enterocyte-like Caco2 cells and comparison with in vivo study. Lett. Appl. Microbiol. 21:146-148.

Del Re, B., B. Sgorbati, M. Miglioli, and D. Palenzona. 2000. Adhesion, autoaggregation and hydrophobicity of 13 strains of Bifidobacterium longum. Lett. Appl. Microbiol. 31:438-442.

FAO/WHO. 2002. Guidelines for the Evaluation of Probiotics in Food. Working Group Report 2002. www.fao.org

Femia, A. P., C. Luceri, P. Dolara, A. Giannini, A. Biggeri, M. Salvadori, Y. Clune, K. J. Collins, M. Paglierani, and G. Caderni. 2002.
Antitumorigenic activity of the prebiotic inulin enriched with oligofructose in combination with the probiotics Lactobacillus rhamnosus and Bifidobacterium lactis on azoxymethane-induced colon carcinogenesis in rats. Carcinogenesis 23:1953-1960.

Gaudier, E., C. Michel, J. P. Segain, C. Cherbut, and C. Hoebler. 2005. The VSL\# 3 probiotic mixture modifies microflora but does not heal chronic dextran-sodium sulfate-induced colitis or reinforce the mucus barrier in mice. J. Nutr. 135:2753-2761.

Gionchetti, P., C. Amadini, F. Rizzello, A. Venturi, and M. Campieri. 2002. Review article: Treatment of mild to moderate ulcerative colitis and pouchitis. Aliment. Pharmacol. Ther. 16:13-19.

Gionchetti, P., K. M. Lammers, F. Rizzello, and M. Campieri. 2005. VSL\#3: An analysis of basic and clinical contributions in probiotic therapeutics. Gastroenterol. Clin. North Am. 34:499-513.

Gotteland, M., O. Brunser, and S. Cruchet. 2006. Systematic review: Are probiotics useful in controlling gastric colonization by Helicobacter pylori? Aliment. Pharmacol. Ther. 23:1077-1086.

Gueimonde, M., L. Jalonen, F. He, M. Hiramatsu, and S. Salminen. 2006. Adhesion and competitive inhibition and displacement of human enteropathogens by selected lactobacilli. Food Res. Int. 39:467-471.

Handley, P. S., D. W. Harty, J. E. Wyatt, C. R. Brown, J. P. Doran, and A. C. Gibbs. 1987. A comparison of the adhesion, coaggregation and cell-surface hydrophobicity properties of fibrillar and fimbriate strains of Streptococcus salivarius. J. Gen. Microbiol. 133:3207-3217.

Kajander, K., K. Hatakka, T. Poussa, M. Farkkila, and R. Korpela. 2005. A probiotic mixture alleviates symptoms in irritable bowel syndrome patients: A controlled 6-month intervention. Aliment. Pharmacol. Ther. 22:387-394.

Kim, H. J., M. I. Vazquez Roque, M. Camilleri, D. Stephens, D. D. Burton, K. Baxter, G. Thomforde, and A. R. Zinsmeister. 2005. A randomized controlled trial of a probiotic combination VSL\#3 and placebo in irritable bowel syndrome with bloating. Neurogastroenterol. Motil. 17:687-696.

McFarland, L. V. 2006. Meta-analysis of probiotics for the prevention of antibiotic associated diarrhea and the treatment of Clostridium difficile disease. Am. J. Gastroenterol. 101:812-822.

Myllyluoma, E., L. Veijola, T. Ahlroos, S. Tynkkynen, E. Kankuri, H. Vapaatalo, H. Rautelin, and R. Korpela. 2005. Probiotic supplementation improves tolerance to Helicobacter pylori eradication therapy-A placebo-controlled, double-blind randomized pilot study. Aliment. Pharmacol. Ther. 21:1263-1272.

Olivares, M., M. P. Diaz-Ropero, N. Gomez, F. Lara-Villoslada, S. Sierra, J. A. Maldonado, R. Martin, J. M. Rodriguez, and J. Xaus. 2006. The consumption of two new probiotic strains, Lactobacillus gasseri CECT 5714 and Lactobacillus coryniformis CECT 5711 , boosts the immune system of healthy humans. Int. Microbiol. 9:47-52.

Ouwehand, A. C., E. Isolauri, P. V. Kirjavainen, S. Tolkko, and S. J. Salminen. 2000. The mucus binding of Bifidobacterium lactis Bb12 is enhanced in the presence of Lactobacillus GG and Lact. delbrueckii subsp. bulgaricus. Lett. Appl. Microbiol. 30:10-13.

Ouwehand, A. C., S. Salminen, and E. Isolauri. 2002. Probiotics: An overview of beneficial effects. Anton. Leeuw. Int. J. G. 82:279-289.

Perdigon, G., C. Maldonado Galdeano, J. C. Valdez, and M. Medici. 2002. Interaction of lactic acid bacteria with the gut immune system. Eur. J. Clin. Nutr. 56:S21-S26.

Pérez, P. F., Y. Minnaard, E. A. Disalvo, and G. L. de Antoni. 1998. Surface properties of bifidobacterial strains of human origin. Appl. Environ. Microbiol. 64:21-26.

Rautava, S., H. Arvilommi, and E. Isolauri. 2006. Specific probiotics in enhancing maturation of IgA responses in formula-fed infants. Pediatr. Res. 60:221-224.

Reid, G., and J. A. Hammond. 2005. Probiotics. Some evidence of their effectiveness. Can. Fam. Physician 51:1487-1493.

Roselli, M., A. Finamore, M. S. Britti, and E. Mengheri. 2006. Probiotic bacteria Bifidobacterium animalis MB5 and Lactobacillus rhamnosus GG protect intestinal Caco-2 cells from the inflammation-associated response induced by enterotoxigenic Escherichia coli K88. Br. J. Nutr. 95:1177-1184. 
Ruas-Madiedo, P., M. Gueimonde, A. Margolles, C. G. de los ReyesGavilan, and S. Salminen. 2006. Exopolysaccharides produced by probiotic strains modify the adhesion of probiotics and enteropathogens to human intestinal mucus. J. Food Prot. 69:2011-2015.

Salminen, S., A. C. Ouwehand, Y. Benno, and Y. K. Lee. 1999. Probiotics: How should they be defined? Trends Food Sci. Technol. 10:107-110.

Santosa, S., E. Farnworth, and P. J. Jones. 2006. Probiotics and their potential health claims. Nutr. Rev. 64:265-274.

Sazawal, S., G. Hiremath, U. Dhingra, P. Malik, S. Deb, and R. E. Black. 2006. Efficacy of probiotics in prevention of acute diarrhoea: A meta-analysis of masked, randomised, placebo-controlled trials. Lancet Infect. Dis. 6:374-382.

Timmerman, H. M., C. J. Koning, L. Mulder, F. M. Rombouts, and A. C. Beynen. 2004. Monostrain, multistrain and multispecies
probiotics-A comparison of functionality and efficacy. Int. J. Food Microbiol. 96:219-233.

Tuomola, E. M., A. C. Ouwehand, and S. J. Salminen. 2000. Chemical, physical and enzymatic pre-treatments of probiotic lactobacilli alter their adhesion to human intestinal mucus glycoproteins. Int. J. Food Microbiol. 60:75-81.

Zocco, M. A., L. Z. dal Verme, F. Cremonini, A. C. Piscaglia, E. C. Nista, M. Candelli, M. Novi, D. Rigante, I. A. Cazzato, V. Ojetti, A. Armuzzi, G. Gasbarrini, and A. Gasbarrini. 2006. Efficacy of Lactobacillus GG in maintaining remission of ulcerative colitis. Aliment. Pharmacol. Ther. 23:1567-1574.

Zoppi, G., M. Cinquetti, A. Benini, E. Bonamini, and E. B. Minelli. 2001. Modulation of the intestinal ecosystem by probiotics and lactulose in children during treatment with ceftriaxone. Curr. Ther. Res. 62:418-435. 